REFERENCES:

[1] Levinson W, Kallewaard M, Bhatia RS, Wolfson D, Shortt S, Kerr EA. 'Choosing Wisely': a growing international campaign. BMJ Qual Saf. 2015;24(2):167-74.

[2] Abeles AM, Abeles M. The clinical utility of a positive antinuclear antibody test result. The American journal of medicine. 2013;126(4):342-8.

Disclosure of Interests: Ai Li Yeo: None declared, Jason Ong: None declared, Kathryn Connelly: None declared, Suong Le: None declared, Ronnie Ptasznik: None declared, Jane Ross: None declared, Eric F. Morand Grant/research support from: AstraZeneca, Bristol Myers Squibb, Janssen, Merck Serono, and UCB, Consultant for: AstraZeneca, Eli Lilly, Janssen, and Merck Serono, Speakers bureau: AstraZeneca, Michelle Leech: None declared DOI: 10.1136/annrheumdis-2019-eular.4517

\begin{tabular}{|l|l}
\hline OP0021 & PREDICTING SEVERE INFECTION IN REPEAT CYCLES \\
OF RITUXIMAB AND EFFECTS OF \\
HYPOGAMMAGLOBULINAEMIA FOR THE TREATMENT \\
OF RHEUMATIC AND MUSCULOSKELETAL DISEASES
\end{tabular}

Md Yuzaiful Md Yusof ${ }^{1,2}$, Edward Vital ${ }^{1,2}$, Damien M. Mcelvenny ${ }^{3}$,

Elizabeth Hensor ${ }^{1,2}$, Sudipto Das ${ }^{1,2}$, Shouvik Dass ${ }^{1}$, Andy C. Rawstron ${ }^{4}$, Maya Buch ${ }^{1,2}$, Paul Emery ${ }^{1,2}$, Sinisa Savic ${ }^{1,2}$. ${ }^{1}$ University of Leeds, Leeds Institute of Rheumatic and Musculoskeletal Medicine, Leeds, United Kingdom; ${ }^{2}$ Leeds Teaching Hospitals NHS Trust, NIHR Leeds Biomedical Research Centre, Leeds, United Kingdom; ${ }^{3}$ University of Manchester, Institute of Population Health, Manchester, United Kingdom; ${ }^{4}$ Leeds Teaching Hospitals NHS Trust, Haematological Malignancy Diagnostic Service, Leeds, United Kingdom

Background: Rituximab (RTX) is effective in treating various rheumatic and musculoskeletal diseases (RMDs). Repeat cycles are often required for disease control but may lead to hypogammaglobulinaemia. Low IgG at baseline has been associated with increased risk of severe infection event (SIE) post-RTX. However, there are limited data on predictors of SIEs in repeat cycles including immunoglobulin levels and B-cell numbers as well as outcomes of hypogammaglobulinaemia.

Objectives: To assess predictors of SIEs in repeat RTX cycles and effects of hypogammaglobulinaemia in terms of SIEs rates, humoral response and its persistence post-cessation of RTX.

Methods: A retrospective study was conducted in the first 700 consecutive ARD patients treated with at least a cycle of RTX in Leeds. IgM, IgA and IgG levels were measured at baseline and 4-6 months after each cycle. For cycles 2-4 (C24 ), predictors for SIEs were analysed using mixed-effects logistic regression analysis.

Results: 550 patients were female, mean(SD) age 56(16) years and median (IQR) disease duration 7.9(3.4-15.0) years. 507(72\%) had RA, 94(13\%) SLE, 49 (7\%) AAV, 14(2\%) inflammatory myopathies, 9(1\%) pSS, 5(1\%) APS, 6(1\%) SSc and $16(3 \%)$ other CTDs. $364(52 \%)$ were biologic-naïve and $514(73 \%)$ were on concomitant DMARDs. Total follow-up: 2880 patient-years (PY). 281 SIEs were recorded in 176 patients (9.8/100 PY). In C1, we had validated that low IgG was predictive of SIE within 12 months of $\mathrm{C} 1$. For cycles 2-4, in multivariable analysis, non-RTX-specific comorbidities [chronic lung OR $(95 \% \mathrm{Cl}) 2.4$ (1.3-4.4), diabetes 2.9 (1.2-6.9), heart failure $6.3(1.4-28.1)$, previous cancer 3.0 (1.3-6.7) and severe infection 6.3 (3.0-13.4)] and RTX-specific variables [higher corticosteroid dose 1.08 (1.02-1.14), higher IgM 1.3 (1-1.7) and longer retreatment time 1.01 (1-1.02)] were associated with increased odds of SIEs, but not B-cell numbers or depletion status. Higher IgG reduced the risk 0.88 (0.8-0.96). Of 103 patients with low IgG for at least 4 months duration, SIEs rates were higher in those with low baseline $\operatorname{lgG}(16.4 \mathrm{PY})$ or acquired it during/post-RTX (21.3 PY) versus those with normal IgG (9.7 PY), 5/8(64\%) had impaired humoral response to pneumococcal and haemophilus following vaccination challenge and only 4/11(36\%) had IgG normalised after switching therapies. Overall, $7(1 \%)$ of the patients required Ig replacement based on recurrent sino-pulmonary SIEs and/or low lgG.

Conclusion: Immunoglobulin should be monitored at baseline and before each RTX cycle to identify patients at risk of SIEs. Vigilance is needed for those with lower $\lg$ as this is a consistent predictor of SIE and may affect infection outcomes when patients are switched to a different bDMARD. For those at risk of SIEs, reduction of corticosteroid dose could reduce risk. Low B-cell numbers were not predictive of SIEs.

Acknowledgement: This research was supported by Octapharma and NIHR (DRF-2014-07-155). The views expressed are those of the author(s) \& not necessarily the NHS, NIHR or DOH.

Disclosure of Interests: Md Yuzaiful Md Yusof: None declared, Edward Vital Grant/research support from: He has received honoraria and research grant support from Roche, GSK and AstraZeneca., Damien M McElvenny: None declared, Elizabeth Hensor: None declared, Sudipto Das: None declared, Shouvik Dass Grant/research support from: Roche and GSK,
Andy C Rawstron: None declared, Maya Buch Grant/research support from: Pfizer LTD, UCB, Consultant for: AbbVie, Eli Lilly, EMD Serono, Pfizer Ltd., Sanofi, Paul Emery Grant/research support from: Pfizer, MSD, AbbVie Bristol-Myers Squibb, Roche, Consultant for: Pfizer, MSD, AbbVie, BristolMyers Squibb, UCB, Roche, Novartis, Gilead,Samsung, Sandoz and Lilly, Sinisa Savic Grant/research support from: Novartis and Sobi DOI: 10.1136/annrheumdis-2019-eular.7573

\section{OP0022 \\ DO MRI-DETECTED EROSIONS IN PATIENTS WITH CLINICALLY SUSPECT ARTHRALGIA PREDICT PROGRESSION TO RHEUMATOID ARTHRITIS? A LONGITUDINAL STUDY}

Fenne Wouters $^{1}$, Xanthe Matthijssen ${ }^{1}$, Debbie Boeters ${ }^{1}$, Robin Ten Brinck ${ }^{1}$, Annette van der Helm - van Mil ${ }^{1,2}$, Ellis Niemantsverdriet ${ }^{1} .{ }^{1}$ Leiden University Medical Centre, Rheumatology, Leiden, Netherlands; ${ }^{2}$ Erasmus University Medical Centre, Rheumatology, Rotterdam, Netherlands

Background: Radiographic joint erosions are a hallmark of Rheumatoid Arthritis (RA). MRI is more sensitive than radiographs in detecting erosions. It is unknown if MRI-detected erosions are predictive for RA-development in patients with Clinically Suspect Arthralgia (CSA).

Objectives: We investigated the prognostic value of MRI-detected erosions (any $\mathrm{MRI}$-erosion, or MRI-erosion characteristics that were recently identified as specific for RA) in CSA.

Methods: Patients presenting with CSA $(n=491)$ underwent contrast-enhanced 1.5T MRI of the wrist, metacarpophalangeal (MCP) and metatarsophalangeal (MTP) joints at baseline. MRIs were scored according to RAMRIS. Presence of any $\mathrm{MRI}$-erosion (erosion score $\geq 1$ ) and RA-specific erosion characteristics as identified previously (grade $\geq 2$ erosions, erosions in MTP5, erosions in MTP1 if aged $<40$ ) were studied with clinically apparent inflammatory arthritis development as outcome (median follow-up 17 months). Analyses were corrected for age, CRP, ACPA and MRI-detected inflammation

Results: Erosions were present in $20.6 \%$ of patients. Presence of erosions was not associated with arthritis development (HR multivariable analysis $0.85(95 \% \mathrm{Cl}$ $0.52-1.40)$ ). Also the different erosion characteristics were not predictive in CSApatients (grade $\geq 2 \mathrm{HR} 1.29$ (95\% Cl 0.40-4.14), erosions in MTP5 HR 0.89 (95\% $\mathrm{Cl} 0.38-2.09)$ and MTP1 if aged <40 HR 0.98 (95\% Cl 0.23-4.21)). MRl-erosions were more prevalent in ACPA-positive than in ACPA-negative patients $(32.3 \%$ versus $18.8 \%, p=0.02$ ). However, no association with arthritis development was observed in both subgroups.

Conclusion: MRI-detected erosions in hands and feet of patients with CSA were not predictive for arthritis development. These data warn against overinterpretation of MRI-detected erosions in CSA.

Disclosure of Interests: Fenne Wouters: None declared, Xanthe Matthijssen: None declared, Debbie Boeters: None declared, Robin ten Brinck: None declared, Annette van der Helm - van Mil Grant/research support from: The research lead ing to these results has received funding from the European Research Council (ERC) under the European Union's Horizon 2020 research and innovation programme (Starting grant, agreement No 714312) and from the Dutch Arthritis Foundation.

The funding source had no role in the design and conduct of the study., Ellis Niemantsverdriet: None declared

DOI: 10.1136/annrheumdis-2019-eular.2991

\section{LB0001 \\ EFFICACY AND SAFETY OF FILGOTINIB FOR PATIENTS WITH RHEUMATOID ARTHRITIS WITH INADEQUATE RESPONSE TO METHOTREXATE: FINCH1 PRIMARY OUTCOME RESULTS}

Bernard Combe $^{1}$, Alan Kivitz ${ }^{2}$, Yoshiya Tanaka ${ }^{3}$, Désirée van der Heijde ${ }^{4}$, Franziska Matzkies ${ }^{5}$, Beatrix Bartok ${ }^{5}$, Lei Ye ${ }^{5}$, Ying Guo ${ }^{5}$, Chantal Tasset ${ }^{6}$, John Sundy ${ }^{5}$, Neelufar Mozaffarian ${ }^{5}$, Robert B.M. Landewé ${ }^{7}$, Sang-Cheol Bae ${ }^{8}$, Edward C. Keystone ${ }^{9,10}$, Peter Nash ${ }^{11}$. ${ }^{1} \mathrm{CHU}$ Montpellier, Montpellier University, Montpellier, France; ${ }^{2}$ Altoona Center for Clinical Research, Duncansville, United States of America; ${ }^{3}$ University of Occupational and Environmental Health Japan, Kitakyushu, Japan; ${ }^{4}$ Leiden University Medical Center, Leiden, Netherlands; ${ }^{5}$ Gilead Sciences, Inc., Foster City, United States of America; ${ }^{6}$ Galapagos NV, Mechelen, Belgium; ; Amsterdam University Medical Center, Amsterdam, Netherlands; ${ }^{8}$ Hanyang University Hospital for Rheumatic Diseases, Seoul, Korea, Rep. of (South Korea); ${ }^{9}$ Mount Sinai Hospital, Toronto, Canada; ${ }^{10}$ University of Toronto, Toronto, Canada; ${ }^{11}$ University of Queensland, St. Lucia, Australia

Background: Filgotinib (FIL) is an orally administered, potent and selective inhibitor of Janus kinase 1 (JAK1) that has shown good efficacy and was well tolerated for treatment of rheumatoid arthritis (RA).

Objectives: To evaluate efficacy and safety of FIL treatment in patients with RA who have had an inadequate response to methotrexate (MTX). 